\title{
Exploration des facteurs influençant la mobilisation des savoirs par une pensée critique chez des étudiantes infirmières bachelières lors de stages cliniques
}

\author{
Kathleen Lechasseur \\ Université Laval, Québec city, Canada, kathleen.lechasseur@fsi.ulaval.ca \\ Ginette Lazure \\ Université Laval, ginette.lazure@fsi.ulaval.ca \\ Louise Guilbert \\ Université Laval, louise.guilbert@fse.ulaval.ca
}

Follow this and additional works at: https://qane-afı.casn.ca/journal

Part of the Curriculum and Instruction Commons, Educational Administration and Supervision Commons, Higher Education Commons, Higher Education and Teaching Commons, and the Nursing Commons

\section{Recommended Citation}

Lechasseur, Kathleen; Lazure, Ginette; and Guilbert, Louise (2014) "Exploration des facteurs influençant la mobilisation des savoirs par une pensée critique chez des étudiantes infirmières bachelières lors de stages cliniques," Quality Advancement in Nursing Education - Avancées en formation infirmière: Vol. 1: Iss. 1, Article 5.

DOI: https://doi.org/10.17483/2368-6669.1003

This Article is brought to you for free and open access by Quality Advancement in Nursing Education - Avancées en formation infirmière. It has been accepted for inclusion in Quality Advancement in Nursing Education - Avancées en formation infirmière by an authorized editor of Quality Advancement in Nursing Education - Avancées en formation infirmière. 


\section{Exploration des facteurs influençant la mobilisation des savoirs par une pensée critique chez des étudiantes infirmières bachelières lors de stages cliniques}

\section{Cover Page Footnote}

Nous remercions les étudiantes ayant bien voulu participer à l'étude sur une base volontaire. En acceptant de partager le vécu de leur expérience en stages, elles ont rendu possible ce projet. Le projet s'inscrit dans le cadre d'études doctorales subventionnées en partie des bourses obtenues de la Fondation de recherche en sciences infirmières du Québec de l'Ordre des infirmières et infirmiers du Québec, du MELSUniversités programme du Ministère de l'Éducation, des Loisirs et des Sports du Québec et du Fonds de soutien au doctorat de l'Université où l'étude s'est déroulée. 


\section{INTRODUCTION}

La complexité des situations de soins s'accroît constamment, entre autres, avec le vieillissement des populations et le perfectionnement technologique. Plus que jamais, le rôle des infirmières en regard de la santé des personnes est mis en évidence. C'est pourquoi elles doivent faire preuve d'une pensée critique pour adapter les divers savoirs conduisant à une vision globale et circonstanciée des situations de soins. Étant novices, les étudiantes tendent toutefois à appliquer leurs savoirs de façon rigide en adoptant une vision fragmentée plutôt que globale. Malgré que leurs difficultés soient bien documentées, aucune étude n'a exploré les facteurs influençant la mise en œuvre d'une pensée critique dans la mobilisation des savoirs des étudiantes lors de stages cliniques. Il demeure donc difficile d'identifier des stratégies pédagogiques favorisant le déploiement d'une pensée critique à la base de la mobilisation des savoirs chez les étudiantes en stages. Ainsi, il importe d'explorer plus en profondeur ce processus tout en adoptant une perspective disciplinaire (Rycroft-Malone, Seers, Tingen, Harvey, Kitson \& Mc Cormack, 2004). L'étude doctorale visait à caractériser le processus de mobilisation des savoirs par une pensée critique chez des étudiantes infirmières bachelières en situation de soins à partir de la théorie Nursing as Caring (Boykin \& Schoenhofer, 1993, 2001, 2006). Plus particulièrement, elle visait à identifier les savoirs interpellés par les étudiantes, le rôle d'une pensée critique dans leur mobilisation ainsi que les facteurs exerçant une influence sur ce processus. Cet article rapporte une partie des résultats de l'étude, soit ceux relatifs aux facteurs d'influence identifiés afin d'offrir des pistes pour guider le choix des stratégies éducatives à privilégier lors des stages cliniques.

\section{CONTEXTE}

\section{Contribution d'une pensée critique}

Les écrits soulignent le rôle primordial d'une pensée critique dans la mobilisation ${ }^{1}$ des savoirs (Argyris, 2000; Ordre des infirmières et infirmiers du Québec [OIIQ], 2009; Paul \& Elder, 2002). De façon évidente, ce processus, intégrant de multiples dimensions, contribue à assurer une pratique infirmière holiste et de qualité (Chan, 2013; Simpson \& Courtney, 2007; Yuan, Williams \& Fan; 2008). Selon une étude panaméricaine, le développement d'une pensée critique chez les futures diplômées représente un des enjeux les plus importants pour la formation infirmière, il importe donc de s'y intéresser (Benner, Tanner \& Chesla, 2009).

Pour bien comprendre sa contribution dans la mobilisation des savoirs, il faut considérer les points de convergence qui se dégagent des nombreuses définitions d'une pensée critique dans la littérature. Au premier plan, sa nature exploratoire et évolutive ressort des écrits. Celle-ci conduit à la reconnaissance des problèmes rencontrés et permet d'amorcer la mobilisation des habiletés cognitives, l'expertise développée et les savoirs (Fesler-Birch, 2005; Schön, 1994). De plus, les écrits font valoir son caractère évaluatif qui permet de poser un jugement sur l'authenticité, la valeur et la pertinence des données recueillies (Edwards, 2003). Une pensée critique fait aussi

\footnotetext{
${ }^{1}$ Le concept mobilisation des savoirs est utilisé selon la définition suivante : « Mobiliser, ce n'est pas seulement « utiliser » ou « appliquer », c'est aussi adapter, différencier, intégrer, généraliser ou spécifier, combiner, orchestrer, coordonner, bref conduire un ensemble d'opérations mentales complexes qui, en les connectant aux situations, transforment les connaissances plutôt que les déplacent ». (Perrenoud, 2002, p.46)
} 
appel à une pensée réflexive et raisonnable (Argyris, 1992; Schön, 1994) en contribuant à examiner la rationalité et la justification des idées ainsi que des actions en fonction des personnes et des contextes (Facione \& Facione, 1996; Forneris \& Peden-McAlpine, 2007). La prise en compte des personnes ne saurait être possible si une pensée critique n'était aussi une pensée responsable ou morale, préoccupée par ce qui est bien et juste pour la personne (Ruiz, 2004). Ainsi, elle vise à déterminer ce qu'il y a lieu de faire ou de croire dans une situation donnée (Ennis, 1985).

Dans une perspective infirmière, une pensée critique contribue à envisager les multiples facettes d'une situation, à identifier et à sélectionner les données pertinentes et suffisantes pour circonscrire les problèmes actuels ou potentiels. Un choix des interventions à privilégier peut ensuite être effectué en considérant les diverses alternatives, leurs conséquences et leurs implications pour les personnes soignées. Les prises de décision se trouvent fondées sur une diversité de savoirs (Edwards, 2003). Fait crucial, le recours à une pensée critique amène l'infirmière à mieux gérer les nombreuses informations et contribue même à faciliter son adaptation face aux perpétuels changements (Paul \& Elder, 2002). À cela, Alfaro-LeFevre (2004) ajoute qu'une pensée critique favorise la connaissance de soi et que sans elle, l'infirmière ne peut résoudre les problèmes complexes rencontrés dans sa pratique. Aussi, une pensée critique permet de prendre conscience de l'influence des émotions dans l'écoute des idées d'autrui et de les réguler (Lafortune \& Robertson, 2005). Enfin, à un niveau métacognitif, une pensée critique favorise l'autorégulation et l'autocorrection des processus de pensée (Paul \& Elder, 2002). Voilà pourquoi une pensée critique constitue une pierre angulaire pour une pratique infirmière optimale contribuant à améliorer la santé des personnes (Chan, 2013; Fesler-Birch, 2005).

\section{Difficultés rencontrées chez les novices}

Les novices rencontrent plusieurs difficultés en milieu de travail (Benner, Tanner \& Chesla, 1992, 2009). Détenant peu d'expérience, leur comportement devient souvent rigide, peu nuancé. Leur démarche s'appuie sur un processus analytique linéaire reposant sur une confiance aveugle aux règles et aux principes théoriques. Face aux données recueillies, les novices se questionnent peu sur leur complétude, leur pertinence et les tendances qui s'en dégagent. L'identification des contradictions entre les données recueillies ou avec celles qui font exception demeure souvent absente (Benner et al., 1992). Les novices éprouvent des difficultés à considérer les diverses éventualités ou alternatives ainsi qu'à détecter une situation clinique exigeant une action immédiate (O’Neill, Dluhy \& Chin, 2005). Elles se centrent principalement sur la tâche à exécuter (Benner et al., 2009). De plus, leur attention porte davantage sur leurs propres besoins plutôt que sur ceux des personnes soignées (Boychuk Duchscher, 2001). Par ailleurs, Benner et al. (1996, 2009) ainsi que Godet \& Chassy (2008) mettent bien en lumière que les novices vivent plusieurs émotions, mais plus particulièrement une forte anxiété pouvant interférer grandement dans leur pratique. Ainsi, plusieurs aspects font obstacle à l'exercice d'une pensée critique chez les novices.

Concernant les étudiantes infirmières, elles présentent les mêmes difficultés que les novices en milieu de travail (Baxter \& Rideout, 2006; Orland-Barak \& Wilhelem, 2005). Leur perception des situations de soins s'avère aussi fragmentée et intègre peu de données contextuelles (Botti \& 
Reeve, 2003). Elles présentent une forte propension à ressentir de l'anxiété et de la crainte en situation de soins (Baxter \& Rideout, 2006; Hutchinson \& Goodin, 2013; Suliman \& Halabi, 2007). Cette anxiété vient entraver l'exercice d'une pensée critique, par exemple, en occasionnant une interprétation inadéquate des informations (Hutchinson \& Goodin, 2013; Suliman \& Halabi, 2007). De nombreux facteurs ont été identifiés comme suscitant de l'anxiété chez les étudiantes. Parmi eux, l'atmosphère régnant dans le milieu de soins représente l'un des facteurs venant le plus moduler les apprentissages des étudiantes en stage (Dale, Leland \& Dale, 2013; Saarikoski, Leino-Kilpi \& Warne, 2002). Lorsque l'équipe soignante ne semble pas les intégrer, elles se sentent rejetées. Elles cherchent alors à se conformer aux exigences et aux attentes du milieu (Dale et al., 2013; Levett-Jones \& Lathlean, 2009; Levett-Jones, Lathlean, Higgins \& McMillan, 2009). La qualité de la relation entre les superviseures et les étudiantes constitue aussi un facteur favorisant leurs apprentissages (Dale et al., 2013), en plus de représenter un déterminant de leur capacité à exercer une pensée critique (Myrick \& Yonge, 2001). En fait, tant la personnalité des superviseures que leurs compétences professionnelles exercent une influence sur le processus de mobilisation des savoirs (Chan, 2002).

Plusieurs études recommandent d'approfondir notre compréhension d'une pensée critique et de sa contribution dans la mobilisation des savoirs en contexte réel de soins ainsi que dans une perspective infirmière (Rycroft-Malone et al., 2004). Elles ne précisent toutefois pas clairement comment se met en œuvre une pensée critique dans la pratique pour permettre une mobilisation des divers savoirs sous-jacents à un soin holiste et de qualité (Gross Forneris, 2005), d'où l'intérêt de s'y pencher. Les trois questions suivantes ont contribué à l'atteinte du but de l'étude doctorale soit:

1. Quels sont les savoirs mobilisés par des étudiantes infirmières bachelières en situation de soins?

2. Comment les savoirs sont-ils mobilisés par une pensée critique chez des étudiantes infirmières bachelières en situation de soins?

3. Quels facteurs exercent une influence dans la mobilisation des savoirs par une pensée critique chez des étudiantes infirmières bachelières en situation de soins?

Rappelons que cet article ne traite que des résultats liés à la troisième question. Les résultats portant sur les savoirs ont été publiés précédemment (Lechasseur, Lazure \& Guilbert, 2011).

\section{MÉTHODE}

L'étude a été réalisée selon une approche qualitative inspirée de la théorisation ancrée (Paillé, 1994; Strauss \& Corbin, 2004). En accord avec la théorie Nursing as Caring (Boykin \& Schoenhofer, 2001, 2006), cette approche a permis de puiser dans l'expérience même des étudiantes infirmières en situation de soins, pour mettre au jour des savoirs interpellés dans leurs actions, faire émerger des processus à la base de leur mobilisation par une pensée critique ainsi que des facteurs venant influencer l'exercice même d'une pensée critique.

\section{Échantillon}

La recherche s'est déroulée dans une université québécoise auprès de seize étudiantes inscrites au programme de formation initiale au baccalauréat en sciences infirmières. Les participantes ont 
été recrutées lors d'une rencontre préparatoire au stage à l'université. Elles devaient avoir réussi plus de la moitié de leur programme et être inscrites dans un cours comportant un stage en chirurgie ou en soins critiques d'une durée de quatorze jours ou portant sur la coordination des soins et la collaboration.

Un échantillon intentionnel a été sélectionné pour tenir compte de contraintes, tel que le calendrier prédéterminé des stages, ayant pu étaler la collecte des données sur une période de temps trop longue dans un contexte d'études doctorales. La saturation théorique a été obtenue à partir de la dixième entrevue (Strauss \& Corbin, 1998). Toutefois, six autres entrevues ont été menées pour rechercher des cas contraires et valider la théorisation émergente.

\section{Méthodes de collecte de données et instruments}

Un questionnaire sociodémographique a été soumis aux étudiantes. L'entretien d'explicitation a été utilisé puisqu'il permet la mise en mots a posteriori d'une action et des processus intellectuels tels que vécus en situation naturelle (Vermersch, 1995). L'entretien portant sur une expérience propre à chaque étudiante, un canevas préétabli regroupant toutes les questions adressées aux participantes ne pouvait être élaboré a priori. Néanmoins, un canevas respectant les phases de l'entretien d'explicitation a été développé. Lors de l'entretien, les étudiantes ont d'abord été guidées vers l'identification d'une situation de soins qu'elles souhaitaient aborder. Nous leur avons demandé ensuite de la décrire globalement, avant de les amener à déterminer un moment plus particulier et significatif dans cette situation, pour focaliser sur l'action réelle et spécifiée (Vermersch, 1995). Des questions descriptives ont porté sur les informations entourant l'action, soit les savoirs, les contextes, les intentions des étudiantes et les évaluations subjectives qu'elles portaient sur la situation pour mettre au jour les savoirs, les processus intellectuels et des facteurs d'influence sur la mobilisation des savoirs par une pensée critique. L'extrait d'un échange avec une étudiante illustre comment ce type d'entretien favorise la mise au jour de la démarche intellectuelle :

P7 : «J'analyse dans ma tête ce qu'elle me dit »

$I$ : "Que fais-tu quand tu analyses? »

$P 7$ : "Je revois l'info que j'ai eue, j'essaie de faire des liens avec mes connaissances. C'est un peu comme la dépendance, c'est quoi la dépendance, c'est quoi la tolérance, je revois, je réfléchis aux définitions que j'ai apprises et j'essaie de voir si chez elle, il y a des choses qui pourraient me dire si ça correspond à ça. »

Une entrevue semi-structurée a permis de valider et de compléter auprès de chacune des étudiantes la reconstitution de la situation de soins décrite lors de l'entretien d'explicitation (Kvale, 1996). Des notes de terrain ont été colligées au cours de la collecte des données afin de garder trace de nos premières impressions sur les aspects factuels et descriptifs des entrevues. Les notes de terrain ont servi à consigner des aspects d'ordre méthodologique, nos réflexions ou nos impressions en cours d'entrevue pour en arriver à des aspects de compréhension ou des pistes d'interprétation. Les entretiens ont tous été enregistrés puis transcrits en verbatim dont le contenu a été validé. La collecte des données a été effectuée sur une période de cinq mois en 2007-2008. 


\section{Analyse des données}

L'analyse des données a été réalisée en suivant la démarche proposée par Paillé (1994). Tout le matériel a été analysé à quatre reprises. Dans un premier temps, nous avons dégagé les actions des étudiantes, notamment les actions mentales pouvant nous fournir des traces d'une pensée critique. Ensuite, l'analyse a porté sur l'identification des savoirs mobilisés. En troisième lieu, nous avons dégagé les conduites faisant preuve d'une pensée critique, pour terminer dans un quatrième temps à l'identification des facteurs d'influence sur le processus à l'étude. Les recommandations de L'Écuyer (1990) ont guidé le processus de catégorisation. Son modèle de catégories mixtes a été retenu. Il comprend certaines catégories préexistantes doublées de catégories à induire. Les catégories préexistantes n'ont pas servi à diriger l'analyse, mais plutôt à la guider pour prévenir l'égarement (L'Écuyer, 1990). Ces catégories préexistantes ne concernaient que les savoirs pouvant être mobilisés en situation de soins, ce qui laissait place à l'émergence d'autres catégories pour caractériser le processus à l'étude. Le logiciel N'Vivo 2 a facilité la saisie et l'analyse des données. Une étape supplémentaire d'analyse a été réalisée, soit la reconstitution de l'action afin de bien en saisir son déroulement tel que vécu (Bachelor \& Joshi, 1986). La lecture de la reconstitution par l'étudiante concernée et la validation par l'entrevue semi-structurée ont permis de certifier leur concordance avec les situations de soins.

\section{Considération d'ordre éthique}

Préalablement à l'étude, le comité d'éthique de l'université concernée a approuvé le protocole de recherche. La chercheure doctorante enseignait à la Faculté où s'est déroulée la recherche. Par conséquent, il était probable qu'elle et les étudiantes se connaissent. Pour éviter une situation de conflit d'intérêts, elle n'a enseigné dans aucun des cours ciblés par la recherche, ni dans aucun des autres cours du programme, et ce, jusqu'à la fin de l'étude. Le consentement volontaire et éclairé, la confidentialité, un équilibre entre les avantages et les inconvénients pour les participantes et une protection contre les conflits d'intérêts ont été assurés par différentes mesures. Avant de signer le formulaire de consentement, les participantes ont été avisées de leur droit de se retirer à tout moment, sans préjudice. La liste des codes attribués aux participantes est gardée sous clé au bureau de la chercheuse et les enregistrements sont conservés dans un ordinateur sécurisé pour 5 ans.

\section{Critères de rigueur scientifique}

Les critères de rigueur proposés pour les recherches qualitatives ont guidé la mise en œuvre de l'étude, c'est-à-dire : la crédibilité, la transférabilité, la fiabilité et la confirmation (Guba, 1981). Par exemple, pour favoriser la crédibilité, les données ont été recueillies à partir du vécu des étudiantes en stage. La triangulation des sources de données va aussi en ce sens (Guba, 1981). La validation de la reconstitution des données obtenues auprès des étudiantes elles-mêmes de même que les moyens mis en place pour assurer la concordance entre le verbatim et la reconstitution de la situation de soins y contribuent également (Blais \& Martineau, 2006; Guba, 1981). Une description détaillée et exhaustive de la démarche suivie et de son contexte a été élaborée pour favoriser la transférabilité des résultats. Plus particulièrement, les procédures d'échantillonnage, les conditions de réalisation des entrevues et notre démarche d'analyse ont été décrites dans la thèse de la première auteure (Lechasseur, 2009). Les commentaires obtenus auprès des étudiantes concernant la synthèse de leurs propos ainsi que le recours à plus d'une méthode de collecte et d'une source de données assurent la fiabilité de la recherche (Bachelor \& Joshi, 1986). Au 
niveau de la confirmation, nous avons, entre autres, fait preuve de transparence en spécifiant l'origine des données et en décrivant le processus d'analyse et d'interprétation des données (Appleton, 1995). L'inclusion de cas contraires et la mise au jour de nos présupposés ainsi que nos prémisses et concepts analytiques y ont aussi contribué (Guba, 1981).

\section{RÉSULTATS}

\section{Caractéristiques de l'échantillon}

Les participantes ( $\mathrm{n}=16)$ à l'étude présentaient des caractéristiques différentes comme le présente le tableau 1. Brièvement, elles étaient âgées en moyenne 22,6 ans (étendue de 21 à 27 ans), la majorité d'entre elles $(n=9)$ avaient une expérience clinique acquise principalement à titre de préposées aux bénéficiaires (PAB). Par contre, la plupart $(n=12)$, n'avaient pas fait de stage comme externe en soins infirmiers au cours de l'été précédent. En ce qui concerne leur formation au niveau du baccalauréat en sciences infirmières, douze $(n=12)$ étudiantes terminaient leur quatrième stage en milieu clinique, d'une durée de quatorze jours, soit en chirurgie. Les autres étudiantes étaient rendues un peu plus loin dans leur formation clinique obligatoire.

Comme le démontre le tableau 2, les situations de soins rapportées étaient variées de même que les caractéristiques des personnes soignées. 
Tableau 1

Les caractéristiques sociodémographiques des étudiantes

\begin{tabular}{|c|c|c|c|c|c|c|c|c|c|}
\hline Participante & Âge & $\begin{array}{l}\text { Expérience } \\
\text { clinique }\end{array}$ & Externat & $\begin{array}{l}\text { Formation } \\
\text { antérieure }\end{array}$ & $\begin{array}{l}\text { Stage : enfants- } \\
\text { adolescents }\end{array}$ & $\begin{array}{c}\text { Stage en } \\
\text { périnatalité }\end{array}$ & $\begin{array}{c}\text { Stage à } \\
\text { l'étranger }\end{array}$ & $\begin{array}{c}\text { Stage : Santé } \\
\text { communautaire }\end{array}$ & $\begin{array}{l}\text { Stage auprès } \\
\text { des aînés }\end{array}$ \\
\hline 1 & 23 & non & non & Sc. de la nature & non & oui & oui & oui & non \\
\hline 2 & 23 & $P A B$ & non & Sc. de la nature & non & oui & oui & oui & non \\
\hline 3 & 21 & $\begin{array}{l}\text { Clinique de } \\
\text { vaccination }\end{array}$ & non & Sc. de la nature & oui & oui & non & non & non \\
\hline 4 & 24 & non & non & Bacc. (santé) & oui & non & oui & non & non \\
\hline 5 & 21 & $P A B$ & non & Sc. de la nature & non & oui & non & non & non \\
\hline 6 & 26 & $\begin{array}{l}\text { Commis } \\
\text { (hôpital) }\end{array}$ & non & Bacc. (santé) & non & oui & oui & non & non \\
\hline 7 & 27 & $P A B$ & non & Commerce & oui & non & oui & non & non \\
\hline 8 & 24 & $P A B$ & non & $\begin{array}{l}\text { Début bacc. } \\
\quad \text { (santé) }\end{array}$ & non & non & non & non & oui \\
\hline 9 & 22 & $P A B$ & oui & Sc. de la nature & oui & oui & non & non & non \\
\hline 10 & 22 & $P A B$ & oui & Sc. de la nature & oui & non & non & non & non \\
\hline 11 & 22 & non & non & Sc. de la nature & non & oui & non & non & non \\
\hline 12 & 21 & non & non & Sc. de la nature & oui & non & non & en cours & non \\
\hline 13 & 21 & non & oui & Sc. de la nature & non & non & non & non & non \\
\hline 14 & 22 & $\begin{array}{c}\text { Préposée à } \\
\text { domicile }\end{array}$ & oui & Sc. de la nature & oui & oui & non & non & non \\
\hline 15 & 22 & non & non & Sc. de la nature & non & oui & oui & oui & non \\
\hline 16 & 21 & non & non & Sc. de la nature & non & oui & non & en cours & non \\
\hline
\end{tabular}


Quality Advancement in Nursing Education - Avancées en formation infirmière, Vol. 1, Iss. 1 [2014], Art. 5

Tableau 2

Les caractéristiques générales des personnes et des situations de soins

\begin{tabular}{|c|c|c|c|c|c|c|}
\hline Situation & Âge & Genre & Problème de santé de la personne & $\begin{array}{c}\text { Stabilité de la } \\
\text { situation/implication }\end{array}$ & Implication de l'étudiante & Milieu \\
\hline 1 & $\begin{array}{l}\text { Entre } 20 \\
\text { et } 30 \text { ans }\end{array}$ & $F$ & Otite & Stable & $\begin{array}{c}\text { Observation et action } \\
\text { directe }\end{array}$ & $\begin{array}{l}\text { Urgence } \\
\text { (triage) }\end{array}$ \\
\hline 2 & $\begin{array}{l}\text { Entre } 60 \\
\text { et } 70 \text { ans }\end{array}$ & $M$ & Chirurgie vésicale (postopératoire immédiat) & Instable & Action directe & Salle de réveil \\
\hline 3 & \pm 70 ans & $M$ & Fistules à l'œesophage, fécalome, désespoir & Stable & Action directe & Chirurgie \\
\hline 4 & \pm 40 ans & $F$ & Chirurgie pour correction de rectocèle & Stable & Action directe & Chirurgie \\
\hline 5 & Âgée & $F$ & Cancer pulmonaire & Fin de vie & Action directe & Médecine \\
\hline 6 & 72 ans & $F$ & $\begin{array}{l}\text { Enclouage de la hanche ( } 2 \text { jours), } \\
\text { confusion, agressivité }\end{array}$ & Instable & Action directe & Chirurgie \\
\hline 7 & 26 ans & $F$ & Pancréatite probable, douleur & Stable & Action directe & Chirurgie \\
\hline 8 & Adulte & $F$ & Cancer de gorge, trachéostomie, anxiété & Stable & Action directe & Chirurgie \\
\hline 9 & \pm 30 ans & $F$ & Hystérectomie, complications vésicales, méfiance & Stable & Action directe & Chirurgie \\
\hline 10 & \pm 65 ans & $F$ & $\begin{array}{c}\text { Détérioration de l'état général, } \\
\text { épisode de bradycardie }\end{array}$ & Instable & Observation & Médecine \\
\hline 11 & $\begin{array}{c}36 \\
\text { semaines }\end{array}$ & $M$ & $\begin{array}{c}\text { Naissance prématurée } \\
\text { (travail prolongé et césarienne) }\end{array}$ & Très instable & Observation & Néonatalogie \\
\hline 12 & \pm 18 ans & $M$ & Pneumothorax et schizophrénie & Instable & Action directe & Chirurgie \\
\hline 13 & \pm 50 ans & $F$ & Choc septique & Très instable & Observation & Soins intensifs \\
\hline 14 & Adulte & $M$ & $\begin{array}{c}\text { Anévrisme aorte abdominale, } M P O C, \\
\text { épisode de dyspnée }\end{array}$ & Instable & Observation & Chirurgie \\
\hline 15 & \pm 30 ans & $M$ & $\begin{array}{c}\text { Dommages cérébraux à la suite } \\
\text { d'une agression physique }\end{array}$ & Instable & Observation & Chirurgie \\
\hline 16 & $?$ & $M$ & $\begin{array}{l}\text { Accident vasculaire cérébral, } \\
\text { perte d'autonomie et aphasie }\end{array}$ & Stable & Action directe & $?$ \\
\hline
\end{tabular}




\section{Facteurs d'influence}

Les facteurs d'influence sur le processus de mobilisation des savoirs par une pensée critique, identifiés au cours de l'étude se regroupent sous deux grandes catégories soient, ceux intrinsèques ou propres aux étudiantes et extrinsèques, soit ceux leur étant externes. Ils sont présentés au Tableau 3.

\section{Facteurs intrinsèques}

Outre leur bagage de connaissances personnel, plusieurs aspects, propres aux étudiantes, influencent la mise en œuvre d'une pensée critique, notamment leur vécu en situation de soins, leurs valeurs et leur conception du rôle infirmier ainsi que leurs traits de personnalité.

Concernant le vécu des étudiantes, la majorité d'entre elles se considèrent des débutantes détenant très peu de connaissances et d'habiletés nécessaires à la pratique infirmière. Il en découle un manque de confiance se traduisant par une forte anxiété qui entraîne une peur de commettre des erreurs pouvant leur faire perdre la face et ainsi être rejetée tant par la personne soignée que par l'équipe soignante. Les étudiantes adoptent alors des comportements qui font valoir d'entrée de jeu leur statut: "Je lui dis que je suis stagiaire et que je vais être son infirmière. ... En disant cela, ça me donne un chapeau. Je compare à un "élève au volant" sur une voiture. (...). Si je suis malhabile..., la personne va être plus "bien ce n'est pas grave, elle est stagiaire...». (P4). Ce manque de confiance en elles, et l'anxiété qui en résulte, viennent entraver leur collecte de données ainsi que diminuer leurs prises d'initiatives et leur autonomie et donc leur capacité de faire preuve de pensée critique. Par contre, il arrive que les émotions, comme la compassion, jouent un rôle de catalyseur et les incitent à s'engager plus activement pour répondre aux besoins de la personne : «(...) j'allais le voir plus souvent, prendre plus soin de lui, être plus alerte au moindre signe de désespoir ou de joie (...). »(P3). Une pensée critique devient plus manifeste par une recherche active d'information, d'alternatives pour répondre aux besoins de la personne. De façon unanime, les étudiantes affirment que les émotions constituent un des principaux facteurs d'influence. Toutefois, elles éprouvent une difficulté à prendre du recul face à leurs émotions, ce qui vient perturber leur capacité de faire preuve de pensée critique: «J'essaie de les dominer, mais c'est parfois difficile. (P8) ».

Les analyses révèlent que les valeurs des étudiantes se transposent dans leur conception du rôle infirmier. En fait, elles constituent un des facteurs d'influence les plus importants sur leurs actions : "Mes valeurs exercent une influence sur mes soins. Pour moi, le respect et la sécurité de la personne sont importants. Je ne ferai pas quelque chose sans savoir. »(P10). Les valeurs fréquemment interpellées concernent le souci de la sécurité et du bien-être physique et psychologique des personnes, la protection de leur vie privée, la préservation de leur dignité, le respect ainsi que leur droit à la vérité. Cependant, comme les étudiantes éprouvent un désir important de se faire accepter, elles tendent à intégrer les valeurs du milieu plutôt que de faire valoir les leurs, même si elles entrent en conflit. Peu d'entre elles font preuve de pensée critique face à un tel conflit. Par contre, certaines se questionnent pour clarifier les valeurs à privilégier dans leur pratique: "Tu établis au fil du temps, tes valeurs, sur quoi tu veux t'orienter (...).» $(P 4)$. Alors, elles adoptent les connaissances transmises par leurs collègues d'une façon plus 
critique, plutôt que conformiste, ce qui dénote l'exercice d'une pensée critique de niveau plus élevé.

Les traits de personnalité des étudiantes émergent comme autre facteur d'influence important. Certains inhibent la mise en œuvre d'une pensée critique. Par exemple, la timidité les amène à demeurer en retrait, à être moins actives. À l'opposé, certains traits stimulent une pensée critique. La curiosité les conduit à s'interroger et à référer à une plus grande diversité de sources d'information. De même, la persévérance et le courage contribuent à la recherche de solutions et d'alternatives plus créatives au bénéfice des personnes.

\section{Facteurs extrinsèques}

Les étudiantes interagissent avec la personne soignée, leur superviseuse, ainsi que toute l'équipe soignante. Or, leurs caractéristiques personnelles, leurs comportements et leurs attitudes influencent la façon dont les étudiantes exercent une pensée critique pour mobiliser leurs savoirs en situation de soins. De plus, des facteurs liés aux situations de soins, liés au contexte scolaire et aux enjeux de pouvoir présents jouent un rôle indéniable.

Concernant les caractéristiques de la personne soignée, les étudiantes mentionnent des aspects d'ordre sociodémographique, notamment le genre et l'âge. En présence d'une personne représentant une clientèle avec laquelle elles se sentent plus à l'aise, il leur est plus facile d'entrer en interrelation et de s'engager. Si elles éprouvent peu d'affinité, il devient plus ardu d'interagir et d'obtenir des informations nécessaires à la compréhension de la situation : « (...) les hommes c'est plus difficile d'aller chercher leurs sentiments (...) et plus âgés c'est plus difficile.» (P7) et ensuite d'intervenir adéquatement. Quant aux caractéristiques personnelles des superviseures, la majorité des étudiantes mentionne que lorsqu'elles démontrent une confiance en soi, leur propre confiance se voit stimulée, en cas contraire elles deviennent plus inquiètes. La passion du soin démontrée par les superviseures est une autre des conditions favorables. Les étudiantes se sentent alors motivées à apprendre, à s'impliquer auprès des personnes soignées. Si les superviseures se montrent peu passionnées, les étudiantes se trouvent freiner dans leurs actions et peuvent même diminuer les contacts avec les personnes soignées limitant alors la recherche d'informations pour comprendre leur situation. Par ailleurs, les étudiantes préfèrent que les superviseures soient expérimentées, tant comme infirmières que comme superviseures. Elles se montrent alors plus aptes à les accompagner dans leurs apprentissages. Les superviseures faisant preuve de rigueur, de questionnement et de qualités relationnelles exercent une influence positive: "Une infirmière qui est compétente dans son domaine et qui fait preuve d'une pensée critique et d'autonomie, ça m'aide. » (P14). Les étudiantes ont besoin de modèles de rôles pour développer leur capacité à exercer une pensée critique et à mobiliser divers savoirs.

En ce qui a trait aux comportements et aux attitudes des personnes que côtoient les étudiantes, ils se regroupent sous quatre catégories interdépendantes : l'ouverture, la confiance, l'acceptation et la collaboration. L'ouverture se manifeste par un intérêt des personnes soignées à partager leur vécu avec les étudiantes et facilite l'établissement d'une relation. Des échanges d'information plus fréquents et plus approfondis surviennent permettant aux étudiantes d'adapter leurs soins sur la base de leurs savoirs et des informations transmises par les personnes. Par exemple, cette étudiante dit : "C'était un patient qui était ouvert aux opportunités, qui me parlait beaucoup de 
lui.., ça m'a beaucoup facilité la tâche. Il me mettait très à l'aise. "(P3). À l'opposé, les étudiantes s'engagent beaucoup moins dans une recherche d'informations active et constante, ou même à intervenir d'une façon personnalisée, lorsque les personnes soignées se montrent peu enclines à partager leur vécu: "Un patient qui ne te parle pas, qui est bête, on ne sera pas portée à aller le toucher, à le questionner. On va faire nos soins et ça va être on sort de la chambre au plus vite. » (P3). L'ouverture et la confiance manifestées par les superviseures et les autres infirmières s'avèrent tout aussi cruciales. Alors, les étudiantes les consultent plus fréquemment pour clarifier leurs incompréhensions ou dissiper leurs doutes ou encore pour mieux comprendre le contexte. Les connaissances des superviseures viennent enrichir leur bagage de connaissances et leur réflexion. En situation inverse, les étudiantes cessent de les questionner et se privent de nouvelles connaissances nécessaires au déploiement d'une pensée critique : Quand tu poses une question et ils sont bêtes, ils répondent vite. On n'a pas tendance à leur parler. » (P9). Il en est de même concernant l'équipe soignante. Par ailleurs, la majorité des étudiantes exprime le besoin que les superviseures et les autres infirmières s'intéressent à elles, non seulement comme stagiaires, mais aussi comme personnes. Elles peuvent ainsi exprimer davantage les nombreuses émotions qu'elles vivent, se sentir écoutées et soutenues : «(...) j'avais besoin de me faire dire que c'est normal que ça me touche que mon patient m'a dit qu'il voulait mourir. »(P3). Ainsi, l'ouverture face aux étudiantes favorise le développement d'une pensée critique par l'approfondissement d'une meilleure connaissance de soi et gestion des émotions.

Concernant la confiance manifestée envers les étudiantes par les personnes soignées, elle suscite un sentiment de reconnaissance de leur compétence et d'acceptation : "Je voyais que j'étais acceptée. J'ai senti que c'était correct que je mette ma main sur son genou. » (P7). En situation contraire, elles éprouvent un sentiment de rejet et de dépréciation. Elles s'investissent très peu alors dans la relation interpersonnelle et dans leurs soins. Les étudiantes apprécient également la confiance démontrée par les superviseures, ce qui vient soutenir la mobilisation de leurs savoirs : "Une relation de confiance avec ma superviseure, ça facilite mon apprentissage, mon autonomie et des actions pertinentes (...). » (P15). En l'absence d'une telle confiance, elles se sentent incompétentes, leur pensée critique de même que leurs savoirs sont alors peu mis à contribution. D'une voix commune, les étudiantes expriment qu'elles n'aiment pas se faire reprendre ou corriger par les superviseures devant les personnes soignées, à moins que ce ne soit nécessaire pour assurer leur sécurité. À leur point de vue, une intervention semblable mine la confiance des personnes envers elle et leur propre confiance en elle, ce qui les conduit à hésiter à faire preuve d'autonomie par la suite pour éviter de porter flanc à la critique.

Au niveau de l'acceptation, elle s'avère tout aussi importante de la part des personnes soignées, des superviseures que par l'ensemble de l'équipe soignante. Il importe que leurs acquis soient pris en considération et que leurs limites soient acceptées : "Oui, d'être dans l'équipe ça m'a vraiment aidée parce que je savais que j'avais des recours puis que j'allais être prise au sérieux. »(P3). Elles disposent alors de ressources supplémentaires venant soutenir leurs apprentissages, notamment en pouvant disposer d'informations auxquelles elles n'auraient pu accéder en l'absence d'une telle acceptation. Dans un tel climat, elles peuvent déployer plus facilement les habiletés intellectuelles à la base d'une pensée critique. La formulation fréquente de rétroactions par les superviseures est primordiale particulièrement lorsqu'elles sont jugées constructives : " J'ai besoin d'être félicitée, j'ai une confiance à construire. "(P11). Une faible manifestation 
d'acceptation provoque une augmentation du stress venant perturber leur fonctionnement global et leur capacité de faire preuve de pensée critique. En ce qui concerne la collaboration, celle des personnes soignées facilite l'établissement d'une relation et d'une complicité avec elles : " $C$ 'est important d'avoir une complicité. Je pouvais faire des choses avec lui. » (P3). De la sorte, elles peuvent obtenir davantage d'information et leurs interventions tiennent davantage compte de la globalité des personnes soignées. Dans les situations où leur collaboration s'avère difficilement obtenue, les échanges interpersonnels deviennent plus difficiles et beaucoup moins fructueux au regard des informations obtenues. Au niveau des superviseures et l'équipe soignante, les étudiantes apprécient pouvoir bénéficier de leur aide et de leur collaboration: «Dans un contexte où je me sens entourée et supportée, ça m'aide à utiliser constructivement mes ressources. (P2) ». Le partage des connaissances, particulièrement expérientielles, vient enrichir celles des étudiantes et leur permet de mieux les adapter en fonction du contexte.

Par ailleurs, plusieurs aspects liés aux situations de soins exercent une influence sur la capacité des étudiantes à faire preuve de pensée critique dans la mobilisation de leurs savoirs en générant un niveau d'anxiété plus ou moins important, soit: la charge de travail et la stabilité des situations de soins ainsi que la variété et la complexité des situations. Au niveau de la charge de travail, si elle dépasse leurs capacités, une augmentation du stress vient perturber leur fonctionnement. Le manque de temps compromet le questionnement et la réflexion, à la base d'une pensée critique, sur leurs actions «Je ne me pose pas trop de questions, car je n'ai pas le temps. » (P11). Si la charge de travail est adéquate et que la condition de santé des personnes est relativement stable, elles se montrent plus enclines à connaître les personnes et parviennent à anticiper leurs besoins et les actions à poser. L'appropriation des procédures techniques dans le temps s'avère un facteur facilitant puisque lorsqu'elles les maîtrisent elles peuvent se centrer sur la personne : "(...) étant donné que je sais comment faire une ponction veineuse maintenant, je prends en compte mon patient. »(P4). En cas contraire, les procédures techniques occupent en grande place, reléguant la personne au second plan.

L'exposition à une variété de situations favorise l'acquisition de nouvelles connaissances, entre autres pratiques, permettant aux étudiantes de mieux comprendre les situations de soins subséquentes et de construire un répertoire d'interventions plus adaptées qu'elles peuvent ensuite intégrées à leur pratique : "Ma monitrice, elle nous a envoyées voir beaucoup d'examens, ça nous a aidées à savoir après comment ça allait avec les patients, comment ils reviennent d'une bronchoscopie. »(P10). Plus spécifiquement, les étudiantes se montrent très préoccupées par l'acquisition d'une meilleure dextérité technique. L'opportunité d'en exercer plusieurs réduit l'anxiété venant perturber leurs démarches cognitives. Par ailleurs, elles se montrent réceptives à adapter leurs façons de faire en fonction des personnes. En regard du choix des situations, les étudiantes recommandent que les superviseures valident préalablement auprès d'elles leur niveau de connaissances et leurs acquis : "Elles doivent vous prendre où vous êtes rendues, ne pas prendre pour acquis que vous savez certaines choses alors qu'il y a des différences [d'un milieu à l'autre]. » (P12). Une forte anxiété survient lorsque les situations auxquelles sont exposées les étudiantes dépassent leurs capacités. Elles réfèrent alors davantage aux connaissances de leurs superviseures plutôt qu'à leurs propres connaissances. Aussi, elles disent apprécier recevoir du soutien face à ces nouveautés : "C'est facilitant quand elle est présente pour me guider lorsque j'accomplis quelque chose de nouveau ou que je ne maîtrise pas bien.» (P12). Cet accompagnement les amène à construire une confiance en elle et ainsi à pouvoir mobiliser 
graduellement leurs savoirs puisqu'elles ont développé une meilleure compréhension de la situation.

Par rapport au contexte scolaire, un seul facteur d'influence émerge, soit le type d'encadrement offert. Le ratio une superviseure pour une stagiaire est privilégié, puisque le temps accordé par les superviseures est supérieur. Une relation plus étroite s'instaure suscitant un plus grand partage de connaissances et une attention plus étroite envers les multiples besoins des étudiantes : "Quand j'avais besoin d'elle, elle était toujours proche, elle était disponible, elle était ouverte, elle répondait à mes questions, ça la dérangeait pas. » (P10). Ces échanges, jugés plus constructifs par les étudiantes, leur permettent de valider leurs connaissances et leur cheminement intellectuel. L'augmentation du ratio étudiantes/superviseure vient altérer ces bénéfices et occasionne des périodes d'inactivité plus fréquentes venant diminuer les opportunités de s'exercer en situation réelle et d'acquérir de l'expérience.

Certains enjeux de pouvoir viennent influencer la capacité des étudiantes à faire preuve de pensée critique et à mobiliser leurs savoirs, notamment leur statut d'étudiantes, le statut d'expert attribué à la superviseure et aux autres infirmières, et le contexte d'évaluation inhérent aux stages cliniques. Les étudiantes se trouvent aussi confrontées aux iniquités entre l'autorité médicale et celle accordée aux infirmières.

Pour les étudiantes, leur statut leur confère un manque de crédibilité auprès de la personne soignée, des superviseures et de l'équipe soignante. Se sentant souvent malhabiles, elles hésitent à intervenir et se retranchent dans une position d'observatrices : "J'ai l'impression qu'elles ne me laissent pas de place pour intervenir. J'aimerais vraiment les aider, agir. Je ne peux pas me rajouter, je suis sûre à 100\% que je vais être de trop. »(P10). Parfois, même si les actions des superviseures viennent en contradiction avec leurs convictions ou leurs connaissances, elles s'abstiennent d'intervenir: "Je me sens coincée dans mon rôle d'étudiante. » (P15). Une peur de commettre des erreurs se fait aussi sentir en lien avec leur inexpérience. Elles cherchent à éviter les reproches, car souvent il leur semble que les erreurs commises sont attribuées spontanément aux étudiantes : "Tu sais le principe que la stagiaire fait des erreurs, qu'eux ils ne sont jamais dans l'erreur. Souvent dans les milieux, (...) aussitôt qu'il y a une erreur, c'est les stagiaires. »(P3).

À l'opposé, elles confèrent un statut d'expert à la plupart des autres infirmières, dont les superviseures, sur la base du nombre d'années d'expérience et aux connaissances qu'elles détiennent : "Quelqu'un qui a plus d'habiletés ou d'expérience, j'ai plus tendance à m'appuyer sur cette personne que sur celle qui en a moins (...). Elle va être plus apte. »(P11). Les superviseures sont perçues comme la figure d'autorité la plus significative et comme : «(...) une source de vérité. Je ne me vois pas dans la position de remettre en doute sa parole. » (P10). Dans cet esprit, les étudiantes tendent à adopter leurs façons de faire tout en les remettant peu en question. Les titres d'emploi exercent aussi une influence : "En plus, elle est assistante. Je ne pense pas que ce soit n'importe qui qu'ils mettent comme assistante. Habituellement, c'est des personnes (...) qui sont une bonne source de connaissances, plus que moi. »(P10).

L'évaluation des apprentissages représente un autre des facteurs d'influence liés aux enjeux de pouvoir. Conscientes du poids du jugement des superviseures dans l'évaluation de leur 
performance et ultimement de leur réussite ou de leur échec en stage, les étudiantes tendent à se modeler étroitement à leurs exigences et à leurs attentes : "Des fois, je ne suis pas d'accord avec ce qu'ils font, mais si ma superviseure me dit de le faire comme ça, je vais le faire...c'est elle la boss. » (P15). Ceci entrave grandement leur capacité de faire preuve de pensée critique et de mobiliser leurs savoirs en fonction des personnes et des situations. Comme il leur semble que l'évaluation des procédures techniques prime, elles y accordent une grande place : Par rapport aux objectifs de stage, il faut faire des techniques. Là, je les avais, c'était marqué manque-les pas. » (P3). Comme elles sont questionnées plus fréquemment sur les besoins physiologiques, les étudiantes y portent davantage attention : "Je m'en vais à la chambre dans le but de prendre les signes vitaux, faire l'évaluation initiale du début de quart de travail. (...) C'est une procédure normale que ma superviseure exigeait.»(P4). Dans cet esprit, il arrive même qu'elles ne répondent pas à des demandes des personnes pour se centrer uniquement sur ce que les demandes des superviseures. Concernant le fait d'être observées par les superviseures dans une optique d'évaluation, il suscite une augmentation importante de leur stress qui peut leur faire perdre leurs moyens et commettre des erreurs inhabituelles : "On dirait que quand on se sent regardée et évaluée ça augmente ton stress (...) et je fais des erreurs (...). On réussirait 100 fois mieux si on n'avait pas ce stress. » (P3). Alors, les étudiantes se centrent sur la tâche à accomplir et elles portent difficilement attention à la personne. De plus, les superviseures accordent une grande attention à la planification du travail. Or, les étudiantes, détenant peu d'expérience, requièrent plus de temps dans l'exécution de leurs actions. Pour parvenir à rencontrer les exigences des superviseures, elles limitent la durée et la fréquence des interactions avec les personnes. Certaines toutefois parviennent à tenir compte de la personne : "Il y a l'histoire qu'il faut faire nos tâches dans le temps voulu, mais il y a un être humain derrière tout ça. » (P5), au risque d'en subir les conséquences dans leur évaluation. Il apparaît donc difficile pour les étudiantes de disposer du temps nécessaire pour exercer une pensée critique et mobiliser leurs savoirs.

L'autorité médicale représente un autre des enjeux de pouvoir à considérer. En effet, les étudiantes perçoivent l'équipe médicale comme figure d'autorité. Les commentaires de la superviseure peuvent contribuer à alimenter cette vision, par exemple : "Une de mes superviseures m'avait dit que les médecins c'étaient des Dieux, qu'il ne fallait pas trop leur parler. » (P3). Par conséquent, la plupart interagissent peu avec les médecins, limitant du même coup les échanges d'information. Il est aussi difficile pour elles de porter un jugement sur leurs recommandations ou même d'ouvrir une discussion avec eux pour mieux comprendre la situation. Exceptionnellement, des étudiantes ont développé une conception intégrant un rapport d'égalité existant entre les deux professions. Il en résulte des échanges facilitant l'obtention d'information pertinente ou l'expression de leurs propres connaissances, notamment celles concernant la personne. Elles peuvent alors faire valoir leur point de vue et exercer une certaine influence sur l'équipe médicale en sollicitant des actions visant le mieux-être de la personne. Ainsi, elles peuvent faire preuve d'une pensée critique, notamment en jouant un rôle d'advocacy, en interpellant sa composante morale. En somme, plusieurs facteurs affectent la mise en œuvre d'une pensée critique chez les étudiantes dans la mobilisation de leurs savoirs. Leur influence varie dans le temps ou d'une situation à l'autre en fonction des personnes impliquées. 
Tableau 3

Certains facteurs d'influence sur le processus de mobilisation des savoirs par une pensée critique

\section{Facteurs intrinsèques aux étudiantes}

- Bagage de connaissances antérieures

- Vécu en situation de soins et émotions

- Valeurs et conception du rôle infirmier

- Traits de personnalité

\section{Facteurs extrinsèques aux étudiantes}

- Caractéristiques sociodémographiques de la personne soignée

- Âge

- Genre

- Caractéristiques propres aux superviseures

- Niveau d'expérience comme infirmières

- Passion du soin

- Niveau d'expérience comme superviseures

- Rigueur

- Qualités relationnelles avec la personne soignée et l'étudiante

- Comportements et attitudes des diverses personnes impliquées

- Ouverture

- Confiance

- Acceptation

- Collaboration

- Liés aux situations de soins

- Charge de travail et stabilité

- Variété et complexité des situations de soins

- Niveau d'appropriation des procédures techniques

- Lié au contexte scolaire

- Type d'encadrement

- Liés aux enjeux de pouvoir

- Statut d'étudiantes

- Statut d'expert des superviseures et des autres infirmières

- Contexte d'évaluation

- Autorité attribuée à l'équipe médicale 


\section{DISCUSSION}

L'étude visait à caractériser la mobilisation des savoirs par une pensée critique en situation de soins, ce qui avait été peu examiné précédemment (Benner et al., 2009). Une des retombées positives de cette recherche s'avère l'identification de facteurs d'influence sur le déploiement de ce processus complexe et itératif.

En ce qui concerne les facteurs intrinsèques, les traits de personnalité des étudiantes, mis en lumière par l'étude, ont été aussi soulevés par plusieurs auteurs (Fesler-Birch, 2005; Paul \& Elder, 2002; Seymour, Kinn \& Sutherland, 2003). À titre d'illustration, la curiosité concoure à une recherche active d'information et d'alternatives contribuant à une pensée critique (Paul \& Elder, 2002). De plus, les résultats corroborent les propos de Beyer (1988) et de Brochu (1999) à l'effet que le niveau de développement d'une pensée critique influence considérablement leur capacité de la mettre en œuvre. En accord avec Edwards (2003), les valeurs personnelles des étudiantes viennent aussi moduler le processus à l'étude.

Par ailleurs, l'étude fait ressortir que plusieurs facteurs d'influence convergent vers la dimension affective, ou le vécu des étudiantes. D'autres auteurs mettent aussi en évidence les interrelations entre les réponses cognitives et émotives (Godet \& Chasy, 2008). Plus particulièrement, comme nos résultats le soulignent l'influence de l'anxiété sur l'apprentissage et l'exercice d'une pensée critique a été aussi constatée par d'autres auteurs, notamment par Hutchinson \& Goodin (2013). Ainsi, les facteurs contribuant à générer ou à augmenter l'anxiété entravent le processus de mobilisation des savoirs par une pensée critique et, à l'inverse, ceux qui favorisent le développement de la confiance contribuent à sa mise en œuvre et à son développement.

À l'instar des écrits consultés (Baxter \& Rideout, 2006; Dale et al., 2013; Evans \& Kelly, 2004; Fornesis \& Peden-McAlpine, 2007; Hutchinson \& Goodin, 2013; St-Pierre, 2007; Suliman \& Halabi, 2007), la plupart des facteurs identifiés dans l'étude sont extrinsèques. Ceux-ci constituent des sources d'anxiété importante entraînant multiples craintes dont celles de commettre des erreurs ou d'être rejetées. Les résultats de la présente étude permettent de discuter plus à fond ces facteurs. Notamment, le fait que certaines caractéristiques personnelles des superviseures jouent un rôle important, par exemple leur expérience à titre d'infirmière et de superviseure. Par leurs propres connaissances, les superviseures peuvent contribuer au développement des savoirs des étudiantes ou apporter les nuances requises pour les contextualiser et soutenir leur pensée critique. La rigueur et la passion du soin rejoignent les dispositions d'une pensée critique et du soin holiste. En ce sens, les superviseures peuvent constituer des modèles de rôle pour les étudiantes. Cependant, lorsque les caractéristiques des superviseures reflètent peu des traits de pensée critique, comme le manque d'ouverture ou d'intérêt à l'autre, elles peuvent nuire au développement d'une pensée critique chez les étudiantes et entraver la mobilisation de leurs savoirs en situation de soins. Les résultats de l'étude apportent également un nouvel éclairage en faisant ressortir que la nature de la relation établie avec les personnes soignées vient moduler le niveau d'ouverture et d'engagement démontré par les étudiantes. Ce constat est en lien avec les fondements de la théorie Nursing as Caring qui fait valoir que le processus de mobilisation des savoirs dépend aussi de la participation de la personne elle-même (Boykin \& Schoenhofer, 1993, 2001, 2006). 
Dans la même veine que d'autres études (Dale et al., 2013; Evans \& Kelly, 2004; Hutchinson \& Goodin, 2013; Myrick \& Yonge, 2001; St-Pierre, 2007; Suliman \& Halabi, 2007), cette recherche met en évidence qu'un manque de collaboration et d'acceptation peut prendre une multitude de formes auxquelles sont très sensibles les étudiantes. Par exemple, le manque d'ouverture face aux lacunes inhérentes à leur statut d'étudiantes ou des critiques non constructives formulées à leur égard peuvent susciter une forte anxiété et une dévalorisation chez elles (Evans \& Kelly, 2004; Levett-Jones \& Lathlean, 2009; Saarikoski et al., 2002).

Une autre contribution de l'étude consiste en l'identification de plusieurs facteurs d'influence liés directement aux situations de soins et qui sont peu explorés par les écrits. Tel que mentionné par plusieurs étudiantes, lorsque la situation de santé des personnes est stable et que la charge de travail qui leur est attribuée tient compte de leurs capacités et de leur niveau de connaissances, leur anxiété est moins importante, ce qui facilite la mobilisation de leurs savoirs et d'une pensée critique. La variété des situations auxquelles les étudiantes sont exposées constitue aussi selon elles un facteur positif, tant qu'elle tient compte de leurs expériences antérieures.

À un autre niveau, la mise au jour des enjeux de pouvoir auxquels font face des étudiantes en situation de soins offre une avancée intéressante. Tel que mentionné par St-Pierre (2007), le fait d'être observées par les superviseures qui se trouvent en position d'autorité est très anxiogène. Ces enjeux viennent s'ajouter aux sources d'anxiété déjà nombreuses lors des stages en milieux cliniques dont plusieurs sont similaires avec celles identifiées dans la littérature (Dale et al., 2013; Forneris \& Peden-McAlpine, 2007; St-Pierre, 2007; Suliman \& Halabi, 2007). Les auteurs y réfèrent en termes de sentiment d'impuissance, de vulnérabilité ou d'incapacité vécus par les étudiantes (St-Pierre, 2007; Suliman \& Halabi, 2007), sans s'attarder aux sources de ces sentiments qui découlent de facteurs extrinsèques aux étudiantes. Deux points n'ont pas été soulevés par les participantes, contrairement à d'autres études (Evans \& Kelly, 2004; St-Pierre, 2007; Suliman \& Halabi, 2007), c'est-à-dire les conditions académiques et les conditions personnelles, ce qui pourrait faire l'objet d'autres recherches. En somme, le processus de mobilisation des savoirs par une pensée critique subit l'influence d'une multitude de facteurs, qui pour la plupart, ne sont pas sous le contrôle des étudiantes. Plusieurs de ces facteurs s'apparentent étroitement à ceux occasionnant de l'anxiété chez les étudiantes qui ont été identifiés antérieurement par d'autres études.

$\mathrm{Au}$ terme de cette étude, certaines recommandations peuvent être formulées. Étant donné l'impact de la personnalité même des étudiantes, des stratégies éducatives contribuant à l'approfondissement chez elles de la connaissance de soi s'avèrent nécessaires pour susciter des prises de conscience favorisant le développement d'une pensée critique. Aussi, vu l'influence considérable de l'anxiété, des stratégies favorisant la reconnaissance, la prise de recul et le contrôle des émotions mériteraient d'être mieux intégrées dans les programmes de formation. De plus, les résultats de l'étude font ressortir clairement que les étudiantes éprouvent le besoin d'être accompagnées, de pouvoir bénéficier du soutien des infirmières et de leur expertise, pour mobiliser leurs savoirs par une pensée critique. Comme les étudiantes ont besoin de sentir qu'elles sont reconnues avec leurs forces et leurs limites, il importe de consolider les mécanismes d'intégration des étudiantes lors des stages cliniques, souvent pris pour acquis, sans être forcément présents. 
Par ailleurs, les superviseures, à titre de modèle de rôle, exercent une influence notable auprès des étudiantes, ce qui vient moduler leur capacité à faire preuve d'une pensée critique et à mobiliser leurs savoirs. Il importe donc de développer des stratégies pour sensibiliser les superviseures à ces enjeux et pour mieux les outiller face aux différentes fonctions liées à la supervision clinique. Aussi, le choix même des situations de soins en fonction des compétences et des connaissances des étudiantes revêt une grande importance. Les superviseures doivent être guidées dans leurs choix tant au niveau de la charge de travail exigée des étudiantes que de la complexité de la situation de soins auxquelles elles peuvent être exposées. Enfin, le contexte d'évaluation dans lequel se trouvent les étudiantes ajoute aux multiples sources d'anxiété présentes et sur la façon d'agir des étudiantes, entre autres, pour se conformer aux attentes. Bien que mentionnés, ces enjeux méritent d'être approfondis par des études ultérieures.

\section{Limites de l'étude}

L'étude s'est déroulée dans une seule université, auprès d'un groupe d'étudiantes uniquement, les résultats ne peuvent être nécessairement transférés dans d'autres contextes académiques, malgré que ce ne soit pas le but d'une étude qualitative. Les situations de soins décrites proviennent d'expériences vécues en milieu hospitalier. D'autres études permettraient d'explorer la transférabilité des résultats à d'autres contextes de soins, par exemple les soins à domicile. L'entretien d'explicitation s'est révélé très riche pour contribuer à la qualité descriptive de nos données. Toutefois, il est possible que d'autres facteurs d'influence n'aient pas été mis au jour d'où l'intérêt de poursuivre des recherches en ce sens. Par ailleurs, au départ il a été considéré que les superviseures de stage faisaient preuve de pensée critique et exerçaient une influence positive en ce sens auprès des étudiantes. Cependant, Tiwari, Avery \& Lai (2003) invitent à la prudence en soulignant qu'il ne faut pas prendre pour acquis que les enseignants s'avèrent tous des penseurs critiques ou en mesure d'évaluer si leurs stratégies d'enseignement favorisent le développement d'une pensée critique. Une autre voie d'exploration s'ouvre donc pour des recherches futures.

\section{CONCLUSION}

L'approche de recherche privilégiée a permis de caractériser le processus de mobilisation des savoirs par une pensée critique chez des étudiantes infirmières bachelières en situation de soins et plus spécifiquement de mettre au jour plusieurs facteurs exerçant une influence sur son déploiement. Certains de ces facteurs sont intrinsèques aux étudiantes, mais la majorité d'entre eux s'avèrent extrinsèques donc pouvant faire l'objet de modifications, de stratégies éducatives ou de mesures de soutien pour les étudiantes et les superviseures encadrant les stages. Des recherches en lien avec les programmes de formation peuvent ainsi être envisagées pour examiner plus étroitement ces facteurs d'influence et leur impact sur le développement et le déploiement d'une pensée critique dans la mobilisation des savoirs chez les étudiantes, nos infirmières de demain. Comme une pensée critique revêt un caractère développemental, l'étude plus détaillée de l'accompagnement qui lui est nécessaire au fil de programmes d'étude en sciences infirmières apparaît cruciale. L'exploration des capacités des enseignants et des superviseures à favoriser le développement d'une pensée critique chez les étudiantes ainsi que leurs propres besoins d'accompagnement s'avère une avenue à privilégier. Par conséquent, les conditions nécessaires aux superviseures pour contribuer significativement aux apprentissages 
des étudiantes en favorisant une mobilisation des savoirs par une pensée critique, et même dans l'équipe soignante, pourraient faire l'objet de recherche pour la formation infirmière. L'intérêt de recourir à l'entrevue d'explicitation dans des recherches futures pour explorer les actions des étudiantes telles que vécues en milieu clinique et ainsi d'explorer leurs processus cognitifs au cœur de l'action justifie de l'utiliser à nouveau comme méthode de collecte de données, bien qu'initialement elle visait l'analyse de pratique.

Les recommandations découlant de cette étude peuvent guider les milieux de formation, les milieux cliniques et les personnes exerçant une influence dans le système de santé pour assurer que des conditions soient mises en place tant en contexte académique, durant les stages, qu'en milieu clinique pour les infirmières novices, afin de favoriser une mobilisation optimale des savoirs par une pensée critique. La personne humaine ne devient pas un penseur critique en un jour et certaines n'y parviennent pas. En vue de contribuer à la formation d'infirmières orientées vers une action responsable et raisonnable pour le bien-être de tous, nos efforts doivent se poursuivre en ce sens. 


\section{RÉFÉRENCES}

Alfaro-LeFevre, R. (2004). Critical thinking and clinical judgment: A practical approach ( $^{\text {rd }}$ ed.). St-Louis: Saunders.

Appleton, J. V. (1995). Analysing qualitative interview data: Addressing issues of validity and reliability. Journal of Advanced Nursing, 22(55), 993-997.

Argyris, C. (1992). Reasoning, learning, and action: individual and organizational. San Francisco: Jossey-Bass.

Argyris, C. (2000). Savoir pour agir: surmonter les obstacles à l'apprentissage organisationnel. (G. Loudière, Trad.). Paris: Dunod.

Bachelor, A., \& Joshi, P. (1986). La méthode phénoménologique de recherche en psychologie: guide pratique. Québec: Les Presses de l'Université Laval.

Baxter, P., \& Rideout, E. (2006). Second-year baccalaureate nursing students' decision making in the clinical setting. Journal of Nursing Education, 45(4), 121-127.

Benner, P., Tanner, C. A., \& Chesla, C. A. (1992). From beginner to expert: Gaining a differentiated clinical world in critical care nursing. Advances in Nursing Science, 14(3), $13-28$.

Benner, P., Tanner, C. A., \& Chesla, C. A. (1996). Expertise in nursing practice: caring, clinical judgment, and ethics. New York: Springer Publishing Company.

Benner, P., Tanner, C., \& Chesla, C. (2009). Expertise in nursing practice: Caring, clinical judgment, and ethics (2 ed.). New York: Springer Publishing Company.

Beyer, B. K. (1988). Developing a thinking skills program. Boston: Allyn and Bacon.

Blais, M., \& Martineau, S. (2006). L'analyse inductive générale: Description d'une démarche visant à donner un sens à des données brutes. Recherches Qualitatives, 26(2), 1-18.

Botti, M. \& Reeve, R. (2003). Role of knowledge and ability in student nurses' clinical decisionmaking. Nursing and Health Sciences, 5(1), 39-49

Boychuk Duchscher, J. E. (2001). Out in the real world. Journal of Nursing Administration's Healthcare Law, Ethics, and Regulation, 31(9), 426-439.

Boykin, A., \& Schoenhofer, S. O. (1993). Nursing as Caring: a model for transforming practice. New York: National league for nursing.

Boykin, A., \& Schoenhofer, S. O. (2001). Nursing as Caring: a model for transforming practice. Sudbury, MA: Jones \& Bartlett and National League for Nursing.

Boykin, A., \& Schoenhofer, S. O. (2006). Nursing as a Caring theory. In M. E. Parker (Ed.), Nursing theories and nursing practice. Philadelphia: F.A. Davis Company.

Brochu, A. C. (1999). Étude de quelques filtres de la pensée critique des futurs enseignants et incidences sur leur formation universitaire. In L. Guilbert, J. Boisvert \& N. Ferguson (Eds.), Enseigner et comprendre (pp. 41-62). Québec: Les Presses de l'Université Laval. 
Chan, D. (2002). Development of the clinical learning environment inventory: Using the theoretical framework of learning environment studies to assess nursing students' perceptions of the hospital as a learning environment. Journal of Nursing Education, 41, 69-75.

Chan, Z.C.Y. (2013). A systematic review of critical thinking in nursing education. Nurse Education Today, 33, 236-240.

Dale, B., Leland, A., \& Dale, J.G. (2013). What factors facilitate good learning experiences in clinical studies in nursing: Bachelor students' perceptions, ISRN Nursing International Scholarly Research Notices, 1-7.

Edwards, S. (2003). Critical thinking at the bedside: A practical perspective. British Journal of Nursing, 12(19), 1142-1149.

Ennis, R. H. (1985). A logical basis for measuring critical thinking skills. Educational Leadership, 43(2), 44-48.

Evans, W., \& Kelly, B. (2004). Pre-registration diploma student nurse stress and coping measures. Nurse Education Today, 24, 473-482.

Facione, N. C., \& Facione, P. A. (1996). Externalizing the critical thinking in knowledge development and clinical judgment. Nursing Outlook, 44, 129-136.

Fesler-Birch, D. M. (2005). Critical thinking and patient outcomes: A review. Nursing Outlook, $53,59-65$.

Forneris, S.G., \& Peden-McAlpine, C. (2007). Evaluation of a reflective learning intervention to improve critical thinking in novice nurses. Journal of Advanced Nursing, 57(4), 410-421.

Godet, F., \& Chassy, P. (2008). Towards an alternative to Benner's theory of expert intuition in nursing: A discussion paper. International Journal of Nursing Studies, 45, 129-139.

Gross Forneris, S. (2005). Improving critical thinking in novice nurses. Thèse de doctorat, Université du Minnesota.

Guba, E. G. (1981). Criteria for assessing the trustworthiness of naturalistic inquiries. Educational Communication and Technology, 29(2), 75-91.

Hutchinson, T.L. \& Goodin, H.J. (2013). Nursing student anxiety as a context for teaching/learning. Journal of Holistic Nursing, 31(1), 19-24.

Kvale, S. (1996). Interviews. An Introduction to Qualitative Research Interviewing. Thousand Oaks, Ca: Sage Publications.

Lafortune, L., \& Robertson, A. (2005). Une réflexion portant sur les liens entre émotions et pensée critique. In L. Lafortune, M. F. Daniel, P. A. Doudin, F. Pons \& O. Albanes (Eds.), Pédagogie et psychologie des émotions: vers la compétence émotionnelle (pp. 62-86). Sainte-Foy: Presses de l'Université du Québec.

Lechasseur, K. (2009). Mobilisation des savoirs par une pensée critique chez des étudiantes infirmières bachelières en situation de soins. Thèse de doctorat, Université Laval, Québec.

Lechasseur, K., Lazure, G. \& Guilbert, L. (2011). Knowledge mobilized by a critical thinking process deployed by nursing students in practical care situations. Journal of Advanced Nursing, 67(9), 1930-1940. 
L'Écuyer, R. (1990). Méthodologie de l'analyse développementale de contenu, méthode GPS et concept de soi. Québec: Presses de l'Université du Québec.

Levett-Jones, T., \& Lathlean, J. (2009). 'Don't rock the boat': Nursing students' experiences of conformity and compliance. Nurse Education Today, 29, 342-349.

Levett-Jones, T., Lathlean, J., Higgins, I., \& McMillan, M. (2009). Staff-student relationships and their impact on nursing students' belongingness and learning. Journal of Advanced Nursing, 65(2), 316-324.

Myrick, F., \& Yonge, O. (2001). Creating a climate for critical thinking in the preceptorship experience. Nurse Education Today, 21(6), 461-467.

O'Neill, E. S., Dluhy, N. M., \& Chin, E. (2005). Modelling novice clinical reasoning for a computerized decision support system. Journal of Advanced Nursing, 49(1), 68-77.

Ordre des infirmières et infirmiers du Québec (2009). Mosaïque des compétences cliniques de l'infirmière. (2e Éd.). Montréal : OIIQ.

Orland-Barak, L. \& Wilhelem D. (2005). Novices in clinical practice settings: student nurses' stories of learning the practice of nursing. Nurse Education Today, 25(6), 455-464

Paillé, P. (1994). L'analyse par théorisation ancrée. Cahiers de recherche sociologique (23), 147181.

Paul, R., \& Elder, L. (2002). Critical thinking: Tools for taking charge of your professional and personal life. Upper Saddle River: Prentice Hall.

Perrenoud, P. (2002). D'une métaphore à l'autre : Transférer ou mobiliser ses connaissances? In J. Dolz \& E. Ollagnier (Eds), L’énigme de la compétence en éducation (pp. 45-60). Paris : de Boeck Université.

Rycroft-Malone, J., Seers, K., Tingen, A., Harvey, G., Kitson, A., \& Mc Cormack, B. (2004). What counts as evidence in evidence-based practice? Journal of Advanced Nursing, 47(1), 81-90.

Ruiz, P.O. (2004). Moral education as pedagogy of alterity. Journal of Moral Education, 33(3), 271-289.

Saarikoski, M., Leino-Kilpi, H., \& Warne, T. (2002). Clinical learning environment and supervision: testing a research instrument in an international comparative study. Nurse Education Today, 22(4), 340-346.

Schön, D. A. (1994). Le praticien réflexif: À la recherche du savoir caché dans l'agir professionnel (J. Heynemand \& D. Gagnon, Trad.). Montréal: Les Éditions Logiques.

Seymour, B., Kinn, S., \& Sutherland, N. (2003). Valuing both critical and creative thinking in clinical practice: narrowing the research-practice gap? Journal of Advanced Nursing, 42(3), 288-296.

Simpson, E., \& Courtney, M. (2007). The development of a critical thinking conceptual model to enhance critical thinking skills in Middle-Eastern nurses: A Middle-eastern experience. Australian Journal of Advanced Nursing, 25(1), 56-63. 
St-Pierre, L. (2007). L'importance du stress vécu par les stagiaires en formation pratique. In L. St-Pierre (Ed.), Se former pour mieux superviser en sciences infirmières (pp. 56-66). Montréal: Beauchemin.

Strauss, A., \& Corbin, J. (1998). Basics of qualitative research: Grounded theory procedures and techniques (2e ed.). Thousand Oaks, CA: Sage.

Strauss, A., \& Corbin, J. (2004). Les fondements de la recherche qualitative: Techniques et procédures de développement de la théorisation ancrée (M.-H. Soulet, Trad.). Fribourg: Academic Press Fribourg.

Suliman, W. A., \& Halabi, J. (2007). Critical thinking, self-esteem, and state anxiety of nursing students. Nurse Education Today, 27, 162-168.

Tiwari, A., Avery, A., \& Lai, P. (2003). Critical thinking disposition of Hong Kong Chinese and Australian nursing students. Journal of Advanced Nursing, 44(3), 298-307.

Vermersch, P. (1995). L'entretien d'explicitation. Issy-les-Moulineaux: ESF Éditeur.

Yuan, H., Williams, B. A., \& Fan, L. (2008). A systematic review of selected evidence on developing nursing students' critical thinking through problem-based learning. Nurse Education Today, 28, 657-663. 